\title{
Usage of the global NM network for assessment of the radiation exposure at flight altitudes
}

\author{
Alexander Mishev* \\ Space Climate Research Unit, University of Oulu, Finland; Sodankylä Geophysical Observatory, \\ University of Oulu, Finland. \\ E-mail: alexander.mishev@oulu.fi

\section{Ilya Usoskin} \\ Space Climate Research Unit, University of Oulu, Finland; Sodankylä Geophysical Observatory, \\ University of Oulu, Finland. \\ E-mail: ilya.usoskin@oulu.fi
}

\begin{abstract}
Exposure to secondary radiation in the atmosphere due to cosmic rays at cruising aviation altitudes is an important topic in the field of space weather. The contribution of galactic cosmic rays to the exposure can be assessed on the basis of models. However, assessment of the dose rate during strong solar particle events (SEPs) is rather complicated, because of their large diversity and stochastic occurrence. Of specific interest are SEPs with energy high enough to produce extensive air showers, which can be registered by ground-based detectors e.g. neutron monitors (NMs), namely ground level enhancements (GLEs). During such events, usually a significant enhancement of the radiation exposure at flight altitudes is observed, specifically over the polar regions. Here, we provide information about derived SEP energy/rigidity spectra using records from the global NM network and the corresponding computed effective doses for several events. A corresponding upgrade of the existing GLE database is provided. We propose to use a convenient proxy based on global NM data in order to assess the effective dose at flight altitude during strong solar particle events.
\end{abstract}

36th International Cosmic Ray Conference - ICRC 2019-

24 July-1 August, 2019

Madison, United States

\footnotetext{
${ }^{*}$ Speaker.
} 


\section{Introduction}

One of the most important problems in the field of space weather is a precise and realistic assessment of the exposure to secondary cosmic ray (CR) radiation, henceforth exposure, at flight altitudes, specifically during strong solar energetic particle (SEP) events [1]. While the exposure due to galactic CRs can be assessed using convenient models and/or proxies [2], realistic computations of the exposure following SEPs are rather complicated, because of the large diversity and stochastic occurrence of the events. Of specific interest are SEPs with energy high enough to produce extensive air showers, whose secondary particles can be registered by ground-based detectors e.g. neutron monitors (NMs), namely ground level enhancements (GLEs) [3, 4]. During such events, usually a significant enhancement of the radiation exposure at flight altitudes is observed, specifically over the polar region $[5,6]$. A realistic information for SEP spectra during such events is crucial to assess the exposure at flight altitudes using a convenient modelling [7].

GLEs are routinely studied using NM records [8]. Historical and recent data are archived at the International GLE database https://gle.oulu.fi [9]. Deployment of NMs at different geographic locations allows one to derive their spectral and angular distribution characteristics, because NMs are sensitive to primary protons arriving from different asymptotic directions. In order to derive the needed spectral characteristics a realistic modelling of the global NM response shall be performed [8]. In this study we derive spectra of several strong GLEs using data from the global NM network (Fig.1). Subsequently, using the derived SEP spectra and model for computation of the effective dose at flight altitudes based on Monte Carlo simulations, we assessed the corresponding exposure during GLEs.

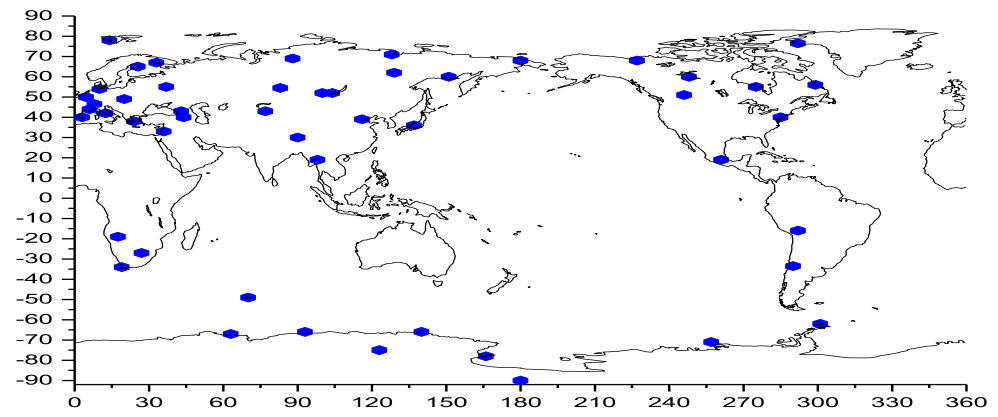

Figure 1: Location map of the present status of the global neutron monitor network.

\section{General description of the procedure for computation of the exposure at flight altitudes}

The procedure for the computation of the exposure at flight altitudes includes several models and steps, the details are given in $[10,11]$. The algorithm includes derivation of SEP spectra using NM data and subsequent application of a model for assessment of the exposure. The analysis of GLEs is performed using a model similar to $[8,12]$, the details given elsewhere $[13,14,15]$. The GLE analysis involves computation of cut-off rigidities and asymptotic viewing directions 
of NMs and optimization of a set of unknown parameters describing specific spectra and angular distribution of the modelled SEPs over the experimental data. Specific shapes can be assumed for the fit of the SEP spectra. Here, we model the spectra of GLE particles by a modified power-law rigidity spectrum $[12,16]$ :

$$
J(P)=J_{0} P^{-(\gamma+\delta \gamma(P-1))}
$$

where $J(P)$ is the differential particle flux with a given rigidity $P$ in $[\mathrm{GV}], \gamma$ is the power-law spectral exponent at rigidity $\mathrm{P}=1 \mathrm{GV}$, accordingly $\delta \gamma$ in $\left[\mathrm{GV}^{-1}\right]$ is the rate of the spectrum steepening. In some cases, specifically during the event onset and initial phase of the event, we model the SEP spectra with an exponential spectrum [16]:

$$
J(P)=J_{0} \exp \left(-P / P_{0}\right)
$$

where $J(P)$ is defined in the same way as in (2.1), accordingly $P_{0}$ is a characteristic proton rigidity. In case of significant steepening $\delta \gamma$, the modified power-law spectrum (2.1) is very similar to exponential shape (2.2). In all cases, the angular distribution of SEPs is modelled with a Gauss like distribution(s):

$$
G(\alpha(P)) \sim \exp \left(-\alpha^{2} / \sigma^{2}\right)
$$

where $\alpha$ is the particles's pitch angle and $\sigma$ corresponds to the width of the distribution(s). The derivation of the angular and spectral characteristics of SEPs is performed over the set of parameters given in Equations (2.1-2.3) by minimizing the difference between the modelled and measured NM responses employing method similar to Levenberg-Marquardt $[17,18]$ with arbitrary regularization $[19,20,21]$.

Hence, using the derived SEP spectra and assuming a conservative approach for their angular distribution - namely an isotropic one [22], on the basis of a model, which demonstrated good agreement with reference data and measurements [23], we can compute the exposure during GLEs [24], considering explicitly the dynamical variation of the derived SEP spectra.

The effective dose rate and/or ambient dose equivalent rate at a given flight altitude $h$ due to primary $\mathrm{CR}$ particles is computed as:

$$
E(h, T, \theta, \varphi)=\sum_{i} \int_{T\left(P_{\text {cut }}\right)}^{\infty} \int_{\Omega} J_{i}(T) Y_{i}(T, h) d \Omega(\theta, \varphi) d T,
$$

where $P_{\text {cut }}$ is the local geomagnetic cut-off rigidity, $\Omega$ is a solid angle determined by the angles of incidence of the arriving particle $\theta$ (zenith) and $\varphi$ (azimuth), $J_{i}(T)$ is the differential energy spectrum of the primary CR at the top of the atmosphere for nuclei of type $i$ (proton or $\alpha$-particle, the latter including effectively heavy nuclei) with kinetic energy $\mathrm{T}$ and $Y_{i}$ is the corresponding yield function. The integration is over the kinetic energy above $T\left(P_{\text {cut }}\right)$, which is defined by $P_{\text {cut }}$ for a nuclei of type $i$. The corresponding effective dose yield function $Y_{i}$ is given by:

$$
Y_{i}(T, h)=\sum_{j} \int_{T^{*}} F_{i, j}\left(h, T, T^{*}, \theta, \varphi\right) C_{j}\left(T^{*}\right) d T^{*}
$$


where $C_{j}\left(T^{*}\right)$ is the coefficient converting the fluence of secondary particles of type $j$ (neutron, proton, $\left.\gamma, e^{-}, e^{+}, \mu^{-}, \mu^{+}, \pi^{-}, \pi^{+}\right)$with energy $T^{*}$ to the effective dose, $F_{i, j}\left(h, T, T^{*}, \theta, \varphi\right)$ is the fluence of secondary particles of type $j$, produced by a primary particle of type $i$ (proton or $\alpha$-particle) with a given primary energy $T$ arriving at the top of the atmosphere from zenith angle $\theta$ and azimuth angle $\varphi$. Note that here, the varying secondary particles spectra and fluence are explicitly considered, since the model is based on Monte Carlo simulations of the CR induced atmospheric cascade, accordingly the conversion coefficients $C_{j}\left(T^{*}\right)$ are derived by Monte Carlo simulations [7, 25]. The same expressions are used for the ambient dose equivalent computation. The full description of the model, look-up tables and application and are given in [23].

\section{Exposure at flight altitudes during selected GLEs}

Using the derived GLE particles spectra and the procedure described in Section 2 - Equations (2.4-2.5), we computed the exposure at typical flight altitudes during strong SEP events, the details are given in [24]. The exposure due to SEPs in most cases is comparable to the contribution of galactic CRs [2], computed here using the force field model [26, 27, 28] and considering the modulation potential as in [29]. In some cases, as during moderately strong GLEs (GLE \#70, GLE \#72), the exposure exceeds 2-5 times the contribution due to GCRs, but does not pose a significant radiation risk [11]. Here, we focus on the two strongest GLEs, namely GLE \#5 on 23 February 1956 and GLE \#69 on 20 January 2005.

GLE \#5 was the largest event observed by the global NM network. It occurred on 23 February 1956, with peak NM count rate increase above $5000 \%$. Using the derived spectra, the details are given in these proceedings, and Equation (2.4) with corresponding yield functions (2.5), we computed the effective dose rate throughout the event.

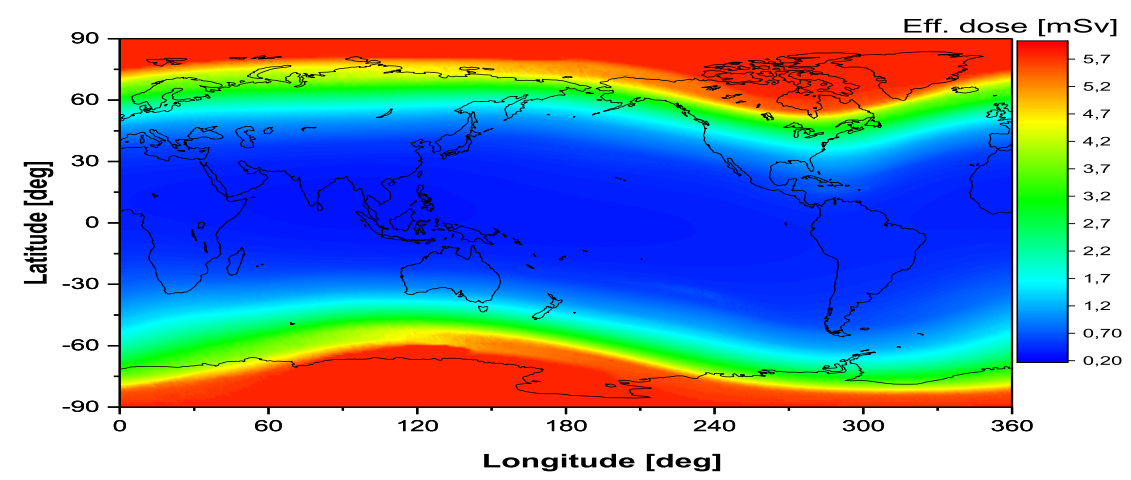

Figure 2: Integrated over the first $3 \mathrm{~h}$ effective dose at altitude of $35 \mathrm{kft}$ during GLE \#5.

As an example, we present the effective dose at altitude of $35 \mathrm{kft}(10668 \mathrm{~m})$ above sea level, integrated over the first $3 \mathrm{~h}$ of the event (Fig.2), accordingly at altitude of $50 \mathrm{kft}$ (15 $240 \mathrm{~m}$ ) (Fig.3). The derived spectra were very hard, specifically during the initial phase of the event, and remained hard during the whole event. This resulted on a significant exposure during the first several hours of the event. One can see that during a typical flight at altitude of $35 \mathrm{kft}$ in a polar region the received dose is comparable to the suggested annual limit for occupational workers of about $6 \mathrm{mSv}$. Note 
that this exposure is considerably greater than that suggested for the population, which is $1 \mathrm{mSv}$. Flight at higher altitude (e.g. $50 \mathrm{kft}$ ), accordingly lead to a considerably greater exposure, about of a factor of 2, which is largely above the annual limit for occupational workers (Fig.3).

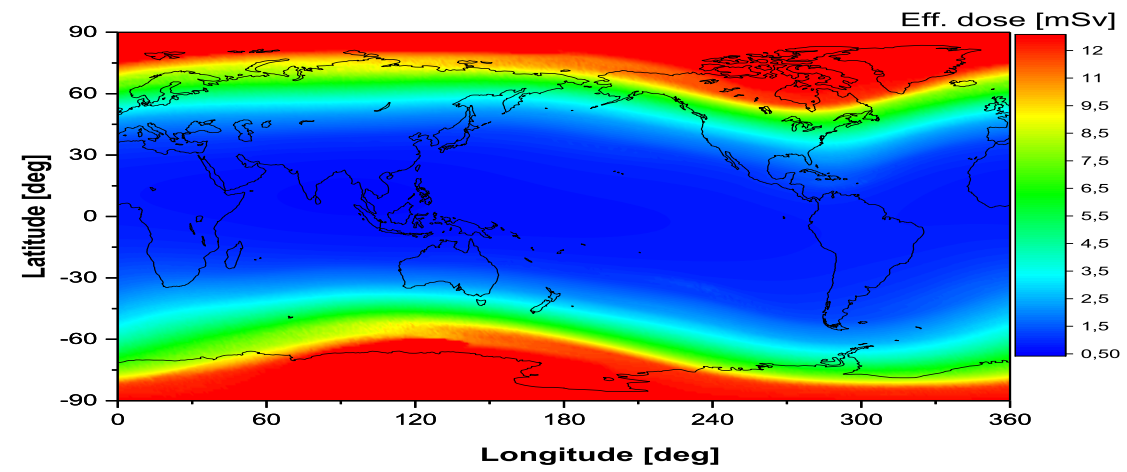

Figure 3: Integrated over the first 3h effective dose at altitude of $50 \mathrm{kft}$ during GLE \#5.

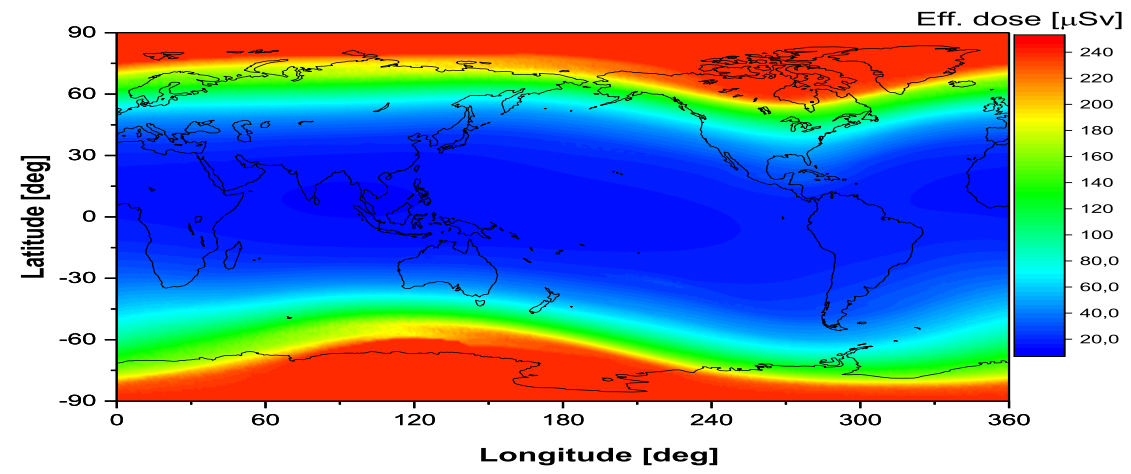

Figure 4: Integrated over the first 3h effective dose at altitude of $35 \mathrm{kft}$ during GLE \#69.

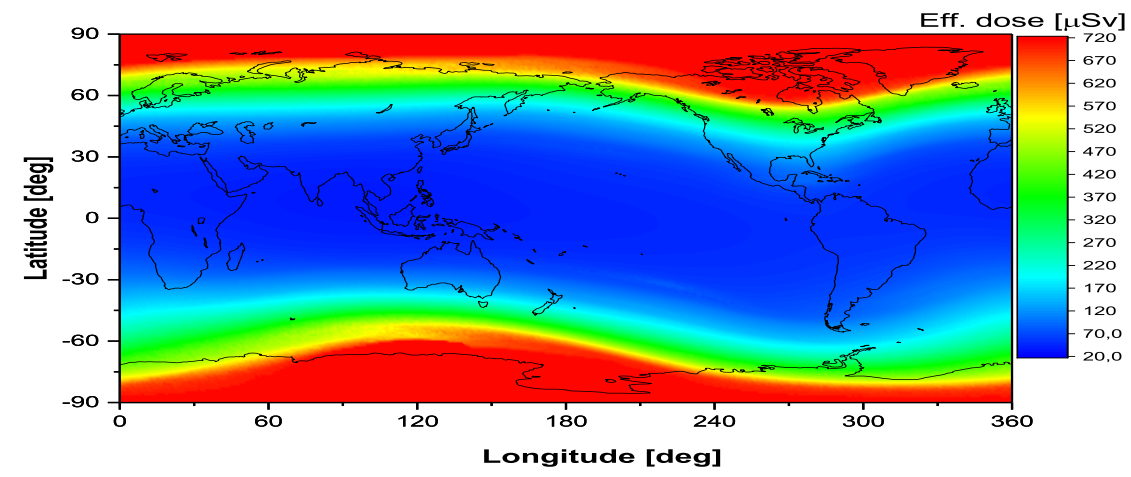

Figure 5: Integrated over the first 3h effective dose at altitude of $50 \mathrm{kft}$ during GLE \#69.

GLE \#69 event on 20 January 2005 occurred during the recovery phase of a Forbush decrease. It was characterized with a pulse like fast NM count rate increase and revealed a large anisotropy, 
since South Pole (SOPO), Terre Adelie (TERA) and McMurdo (MCMD) stations registered significantly greater NM count rate increases than the other NMs. Our analysis depicted a very complex picture - we observed SEPs arriving within two fluxes, the details are given in these proceedings. A very narrow flux with a very hard spectrum, was responsible for the giant NM count rate increases at SOPO, TERA and MCMD NMs and a second flux characterized by a softer, but still hard spectrum, led to the count rate increases of the bulk of the stations. Therefore, the exposure was computed as superposition of the contributions of the two SEP fluxes and GCRs. The derived SEP spectra were hard with high peak intensity, specifically during the event initial phase. The spectra constantly softened during the event, accordingly the particle flux decreased. This resulted on relatively significant exposure during the event onset, but for a short period of time. Similarly to the previous case, we computed the exposure at altitude of $35 \mathrm{kft}$ (Fig.4) and $50 \mathrm{kft}$ (Fig.5), integrated over the first $3 \mathrm{~h}$ of the event. The exposure was significant, about $250 \mu \mathrm{Sv}$, at altitude of $35 \mathrm{kft}$ above sea level. However, it is below the annual limit, but should be considered for air crew and/or frequent travellers. At higher altitude, the exposure considerably increased. According to our computation it was about $700 \mu \mathrm{Sv}$, which could lead to an accumulated dose comparable to the annual limit.

\section{Conclusion}

In this study we demonstrated the possibility to use the global NM network for computation of the exposure at typical commercial jet flight altitudes. Using an appropriate model and reconstructed GLE particles rigidity spectra we assessed the exposure for crew members/passengers at two altitudes, namely 35 and $50 \mathrm{kft}$ above sea level, assuming a conservative isotropic approach of the GLE particles angular distribution. Here, we studied the two strongest GLEs, in order to assess nearly worst case scenario of maximal exposure. It was shown, that at altitude of $50 \mathrm{kft}$ above sea level, the exposure during GLE \#5 is significantly greater that the annual general public limit of 1 $\mathrm{mSv}$ and comparable with this limit during GLE \#69. At lower altitudes, the exposure diminish, because the softer GLE particles spectra, specifically in the case of GLE \#69. Therefore, the two strongest GLE events, led to an important radiation issue. The results presented in this study, allow one to assess the radiation risk at flight altitudes during strong and extreme SEP events [30] and demonstrate the usage of the global NM network for space weather applications, namely as a proxy, for details see [2].

\section{Acknowledgements}

This work was supported by the Academy of Finland (project 307411, Center of Excellence ReSoLVE). We acknowledge the International Space Science Institute support to International Team 441: High EneRgy sOlar partICle Events Analysis (HEROIC). We acknowledge neutron monitor database (NMDB) and all the colleagues and PIs from the neutron monitor stations, who kindly provided the data used for the purposes of this study. 


\section{References}

[1] A. Mishev and P. Jiggens, Preface to measurement, specification and forecasting of the solar energetic particle (SEP) environment and ground level enhancements (GLEs), J. Space Weather Space Clim. 9 (2019) E1.

[2] A. Mishev, S. Tuohino and I. Usoskin, Neutron monitor count rate increase as a proxy for dose rate assessment at aviation altitudes during GLEs, J. Space Weather Space Clim. 8 (2018) A46.

[3] M. A. Shea, I. Zaljubovsky, M. Wada and A. Inoue, A suggested standardized format for cosmic ray ground-level event data, in Proc. of 19th ICRC La Jolla, USA, 11 - 23 August 1985, vol. 5, pp. 510-513, 1985.

[4] S. Poluianov, I. Usoskin, A. Mishev, A. Shea and D. Smart, GLE and sub-GLE redefinition in the light of high-altitude polar neutron monitors, Solar Physics 292 (2017), no. 11176.

[5] F. Spurny, I.Votockova and J. Bottollier-Depois, Geographical influence on the radiation exposure of an aircrew on board a subsonic aircraft, Radioprotection 31 (1996), no. 2 275-280.

[6] F. Spurny, T.Dachev and K. Kudela, Increase of onboard aircraft exposure level during a solar flare, Nuclear Energy Safety 10 (2002), no. 48 396-400.

[7] M. Pelliccioni, Overview of fluence-to-effective dose and fluence-to-ambient dose equivalent conversion coefficients for high energy radiation calculated using the fluka code, Radiation Protection Dosimetry 88 (2000), no. 4 279-297.

[8] M. Shea and D. Smart, Possible evidence for a rigidity-dependent release of relativistic protons from the solar corona, Space Science Reviews 32 (1982) 251-271.

[9] I. Usoskin, A. Ibragimov, M. Shea and D. Smart, Database of ground level enhancements (gle) of high energy solar proton events, Proceedings of Science, Proc. of 34th ICRC Hague, Netherlands, 30 July - 6 August 2015 (2015) 054.

[10] A. Mishev, S. Poluianov and I. Usoskin, Assessment of spectral and angular characteristics of sub-GLE events using the global neutron monitor network, Journal of Space Weather and Space Climate 7 (2017) A28.

[11] A. Mishev and I. Usoskin, Assessment of the radiation environment at commercial jet-flight altitudes during GLE 72 on 10 September 2017 using neutron monitor data, Space Weather 16 (2018), no. 12 1921-1929.

[12] J. Cramp, M. Duldig, E. Flückiger, J. Humble, M. Shea and D. Smart, The October 22, 1989, solar cosmic enhancement: ray an analysis the anisotropy spectral characteristics, Journal of Geophysical Research 102 (1997), no. A11 24 237-24 248.

[13] A. Mishev, L. Kocharov and I. Usoskin, Analysis of the ground level enhancement on 17 May 2012 using data from the global neutron monitor network, Journal of Geophysical Research 119 (2014) 670-679.

[14] A. Mishev and I. Usoskin, Analysis of the ground level enhancements on 14 July 2000 and on 13 December 2006 using neutron monitor data, Solar Physics 291 (2016), no. 4 1225-1239.

[15] A. Mishev, I. Usoskin, O. Raukunen, M. Paassilta, E. Valtonen, L. Kocharov and R. Vainio, First analysis of GLE 72 event on 10 September 2017: Spectral and anisotropy characteristics, Solar Physics 293 (2018) 136. 
[16] E. Vashenyuk, Y. Balabin, J. Perez-Peraza, A. Gallegos-Cruz and L. Miroshnichenko, Some features of the sources of relativistic particles at the sun in the solar cycles 21-23, Advances Space Research 38 (2006), no. 3 411-417.

[17] K. Levenberg, A method for the solution of certain non-linear problems in least squares, Quarterly of Applied Mathematics 2 (1944) 164-168.

[18] D. Marquardt, An algorithm for least-squares estimation of nonlinear parameters, SIAM Journal on Applied Mathematics 11 (1963), no. 2 431-441.

[19] A. Tikhonov, A. Goncharsky, V. Stepanov and A. Yagola, Numerical Methods for Solving ill-Posed Problems. Kluwer Academic Publishers, Dordrecht, 1995.

[20] S. Mavrodiev, A. Mishev and J. Stamenov, A method for energy estimation and mass composition determination of primary cosmic rays at the Chacaltaya observation level based on the atmospheric Cherenkov light technique, Nuclear Instruments and Methods in Physics Research, Section A: Accelerators, Spectrometers, Detectors and Associated Equipment 530 (2004), no. 3 359-366.

[21] R. Aster, B. Borchers and C. Thurber, Parameter estimation and inverse problems. Elsevier, New York, 2005.

[22] K. Copeland, H. Sauer, F. Duke and W. Friedberg, Cosmic radiation exposure of aircraft occupants on simulated high-latitude flights during solar proton events from 1 January 1986 through 1 January 2008, Advances in Space Research 42 (2008), no. 6 1008-1029.

[23] A. Mishev and I. Usoskin, Numerical model for computation of effective and ambient dose equivalent at flight altitudes: Application for dose assessment during gles, Journal of Space Weather and Space Climate 5 (2015), no. 3 A10.

[24] S. Tuohino, A. Ibragimov, I. Usoskin and A. Mishev, Upgrade of GLE database: Assessment of effective dose rate at flight altitude, Advances in Space Research 62 (2018), no. 2 398-407.

[25] N. Petoussi-Henss, W. Bolch, K. Eckerman, A. Endo, N. Hertel, J. Hunt, M. Pelliccioni, H. Schlattl and M. Zankl, Conversion coefficients for radiological protection quantities for external radiation exposures, Annals of the ICRP 40 (2010), no. 2-5 1-257.

[26] L. Gleeson and W. Axford, Solar modulation of galactic cosmic rays, Astrophysical Journal 154 (1968) 1011-1026.

[27] R. Burger, M. Potgieter and B. Heber, Rigidity dependence of cosmic ray proton latitudinal gradients measured by the Ulysses spacecraft: Implication for the diffusion tensor, Journal of Geophysical Research 105 (2000) 27447-27445.

[28] I. Usoskin, K. Alanko-Huotari, G. Kovaltsov and K. Mursula, Heliospheric modulation of cosmic rays: Monthly reconstruction for 1951-2004, Journal of Geophysical Research 110 (2005), no. A12108.

[29] I. Usoskin, G. Bazilevskaya and G. Kovaltsov, Solar modulation parameter for cosmic rays since 1936 reconstructed from ground-based neutron monitors and ionization chambers, Journal of Geophysical Research 116 (2011) A02104.

[30] P. Jiggens, C. Clavie, H. Evans, T. O’Brien, O. Witasse, A. Mishev, P. Nieminen, E. Daly, V. Kalegaev, N. Vlasova, S. Borisov, S. Benck, C. Poivey, M. Cyamukungu, J. Mazur,

D. Heynderickx, I. Sandberg, T. Berger, I. Usoskin, M. Paassilta, R. Vainio, U. Straube, D. Müller, B. Sánchez-Cano, D. Hassler, J. Praks, P. Niemelä, H. Leppinen, A. Punkkinen,

S. Aminalragia-Giamini and T. Nagatsuma, In situ data and effect correlation during september 2017 solar particle event, Space Weather 17 (2019), no. 1 99-117. 